\title{
Transmission des Filarioses
}

\section{Limitation des passages des microfilaires ingérées}

vers l'hémocèle du vecteur; interprétation *

par O. BAIN

Laboratoire de Zoologie (Vers) Centre Muraz - Section Onchocercose

Bobodioulasso, Haute-Volta

Résumé.

Summary.

Introduction.

Chapitre I : Mise en évidence d'un nombre critique de microfilaires ingérées (seuil de limitation) au-dessus duquel le pourcentage de réussite des microfilaires diminue ; existence de microfilaires aptes et de microfilaires inaptes à traverser la paroi stomacale du vecteur.

Chapitre II : Remarques sur la distribution de fréquence des vecteurs en fonction du nombre des microfilaires ingérées ; coexistence de deux modes de répartition des microfilaires chez le donneur.
A. - Wuchereria - Anopheles.
B. - Wuchereria - Aedes.
C. - Setaria - Aedes.
D. - Onchocerca - Simulium.

Chapitre III : Les vecteurs, dont les prises de microfilaires sont comprises entre 0 et le seuil de limitation, correspondent à ceux qui ont une distribution poissonienne.

Chapitre IV : La possibilité, pour un même vecteur, d'ingérer des « microfilaires + » ou des « microfilaires — » pourrait s'expliquer par l'existence de deux modes de piqûre.

Conclusions. Mesure du degré d'adaptation Filaire-vecteur. Santé.

* Ces recherches ont pu être effectuées grâce à une subvention de l'Organisation Mondiale de la 


\section{Résumé}

Seule une fraction des microfilaires ingérées par le vecteur passe dans l'hémocèle et pəut se développer en stades infestants. Il existe deux types de relations numériques entre microfilaires ingérées et microfilaires passées dans l'hémocèle :

1) la proportion des microfilaires passées dans l'hémocèle augmente en fonction du nombre des microfilaires ingérées: c'est la facilitation, observée chez le couple Wuchereria bancrofti Anopheles gambiae $A$ (457 @ disséquées) et étudiée dans un article précédent.

2) le nombre des microfilaires passées dans l'hémocèle est proportionnel au nombre des microfilaires ingérées pour les faibles prises de microfilaires; puis, quand le repas est plus riche en microfilaires, il atteint un chiffre limite: c'est la limitation, mise en évidence chez 3 couples: Wuchereria bancrofti - Aedes aegypti (100 q disséquées), Setaria labiatopapillosa - A. aegypti (357 q disséquées), Onchocerca volvulus - Simulium damnosum (550 q disséquées).

\section{1) Interprétation du phénomène de limitation.}

Aucune image histologique ne permet de l'expliquer. Par contre, un ensemble de faits, issus d'analyses des résultats numériques, conduit à une hypothèse qui fait intervenir à la fois l'état physiologique et le mode de dispersion des microfilaires chez l'hôte vertébré.

a) Chez les 3 couples Filaire-vecteur, présentant le phénomène de limitation, on constate que le nombre des microfilaires passées dans l'hémocèle est proportionnel au nombre des microfilaires ingérées jusqu'à un nombre critique de microfilaires ingérées, que nous appelons le seuil de limitation (il est de 25 microfilaires chez Wuchereria - Aedes, 6-9 microfilaires chez Setaria - Aedes et 3 microfilaires chez Onchocerca - Simulium); au-dessus de ce seuil, la proportion décroît. Tout se passe donc comme si le vecteur qui fait de petits repas de microfilaires prélevait des microfilaires physiologiquement aptes à traverser la paroi stomacale (microfilaires + ) et que le vecteur qui fait des repas riches en microfilaires prélevait des microfilaires inaptes à traverser la paroi stomacale (microfilaires -).

b) L'analyse de la distribution des vecteurs en fonction du nombre de microfilaires ingérées montre qu'une partie des femelles semble prélever ses repas dans un milieu où les microfilaires ont une dispersion homogène (distribution des femelles selon une courbe de Poisson) et qu'une autre partie des femelles semble prélever des microfilaires dans un autre milieu, où elles sont dispersées de façon anarchique et en densités plus fortes (dis- 
tribution des femelles « non poissonienne »). Les deux ensembl:s de distributions se recouvrent légèrement, à leur contact.

c) Si l'on marque en abcisses sur les histogrammes de distribution des femelles le seuil de limitation, on constate qu'il correspond à la séparation entre la population des femelles à distribution essentiellement poissonienne et le reste de la population, comprenant l'aire de recouvrement plus la distribution résiduelle. Cette corrélation (retrouvée trois fois) signifie pour nous que la population des femelles chez laquelle le pourcentage de réussite des microfilaires est élevé correspond à la population des femelles qui a une distribution poissonienne. Ainsi, les microfilaires dispersées de façon homogène semblent correspondre aux microfilaires aptes à traverser la paroi stomacale (microfilaires + ).

d) La possibilité, pour un même vecteur, d'ingérer des « microfilaires + » ou des «microfilaires — » semble être étayée par la mise en évidence chez $A$. aegypti de deux mécanismes de piqûre (Gordon et Lumsden, 1939).

\section{2) Conséquences.}

L'étude de la distribution des veoteurs en fonction du nombre des microfilaires ingérées apparaît comme un moyen de mesurer le degré de coaptation du couple Filaire-vecteur. Dans le cas d'une parfaite adaptation, seules les «microfilaires + doivent être ingérées, ce qui se traduira par une distribution poissonienne du vecteur. Le couple Dipetalonema perstans - Culicoides austeni semble réaliser cet équilibre (chiffres publiés par Nicholas et Kershaw, 1954).

Au contraire, chez Onchocerca - Simulium (souche voltaïque), la plupart des Simulies ingèrent des microfilaires inaptes à traverser la paroi stomacale. Mais ce même couple, en région forestière du Cameroun ne paraît pas présenter un tel degré d'inadaptation (chiffres de Duke et Lewis, 1964). L'adaptation Filaire-vecteur, qui est le résultat d'une longue suite de sélections, varie d'une région géographique à l'autre.

\section{Summary}

From all microfilariae ingested by the vector, only a fraction of the total lot is able to reach the hoemocel and develop into infective stages. The numerical relations between the ingested lot and the hoemocel-reaching microfilariae are of two kinds.

1) The percentage of the hoemocel-reaching microfilariae increases with the number of ingested parasites: it is the facilitation phenomenon which has been observed in the couple Wuche- 
reria bancrofti - Anopheles gambiae A (457 dissected females) and studied in a previous paper.

2) The number of microfilariae that are able to reach the hoemocel is proportional to the number of ingested microfilariae during a slight meal of microfilariae; but when the rate of ingestion increases (heavier meal of microfilariae) this number is limited: it is the limitation phenomenon which has been observed in three couples: Wuchereria bancrofti - Aedes aegypti (100 dissected females), Setaria labiatopapillosa - A. aegypti (357 dissected females), Onchocerca volvulus - Simulium damnosum (550 dissected females).

1) Interpretation of the limitation phenomenon.

No explanation could be found from histological observations, but an analysis of the numerical results leads to an hypothesis which takes into account both the physiological state and the distribution pattern of the microfilariae in their vertebrate host.

a) In the three couples Filaria-vector, which display the limitation phenomenon, it was observed that the number of microfilariae, which have reached the hoemocel, is proportional to the number of ingested microfilariae up to a critical figure, which we name the limitation point (25 microfilariae in Wuchereria - Aedes, 6-9 microfilariae in Setaria - Aedes and 3 microfilariae in Onchocerca - Simulium); above this point, the proportion decreases. Everything seems to occur as if the vector during a slight meal was able to select some microfilariae which are physiologically fitted for crossing the stomach wall (microfilariae +), and, on the contrary, as if a vector having a heavy meal of microfilariae could possibly ingest an amount of microfilariae which are unable to cross the stomach wall (microfilariae -).

b) An analysis of the vector distribution according to the number of ingested microfilariae has shown that some of the females seem to feed on a medium microfilariae are evenly distributed (distribution of females according to Poisson's curve), while other females seem to feed on another medium where microfilariae are disorderly distributed and with higher densities (distribution of females not following the Poisson curve). The two distribution areas are slightly overlapping.

c) If we put on the abscissa of the distribution of the females' histograms the values of the limitation points, we observe that it is in correlation with the disjunction between the population of females strictly following the Poisson curve, and the remaining lot of the population including the overlapped area plus the residual distribution area. To our opinion this correlation, which has been observed three times, indicates that the female population where the percentage of successful microfilariae is high, is 
significant also of a female population which is distributed according to the Poisson curve. Thus the evenly distributed microfilariae seem to include the microfilariae which are able to cross the stomach wall (microfilariae +).

d) The possibility for the same vector to ingest either microfilariae + or microfilariae - seems to be supported by the fact that two different stinging processes have been observed on Aedes aegypti (Gordon et Lumsden, 1939).

\section{2) Consequences.}

The study of the vector distribution related to the number of ingested microfilariae seems to be an efficient tool when attempting to measure the coaptation coefficient in the couple Filariavector. In the case of a complete adaptation, the microfilariae + only are supposed to be ingested and this is shown by a vector distribution following Poisson's curve. This is demonstrated by the couple Dipetalonema perstans - Culicoides austeni (figures published by Nicholas and Kershaw, 1954).

On the contrary in Onchocerca - Simulium (Upper Volta strain) most of the Simulies ingest microfilariae which are unable to cross the stomach wall. But in the Cameroun forest this couple displays a lower inadaptation possibility (figures given by Duke et Lewis, 1964). The adaptation Filaria-vector which is the result of some long and successive selections is submitted to variations according to different geographic areas.

\section{Introduction}

Dans deux articles précédents (Brengues et Bain, sous presse, et Bain et Brengues, sous presse) nous avons mis en évidence deux types de relations numériques entre les microfilaires ingérées et celles qui passent dans l'hémocèle: la facilitation et la limitation.

Le détail des conditions expérimentales et des données numériques, ainsi qu'un exposé préliminaire de nos conclusions, a été distribué sous forme de thèse polycopiée (1971).

Dans la facilitation, la proportion des microfilaires quittant l'estomac augmente avec le nombre des microfilaires ingérées. Un tel phénomène, observé chez le couple Wuchereria bancrofti-Anopheles gambiae A semble être dû à une forte réaction de l'épithélium digestif au passage des microfilaires: les cellules atteintes sont hypertrophiées et constituent des sites nouveaux très favorables à des passages ultérieurs de microfilaires.

Dans la limitation, la proportion des microfilaires quittant l'estomac diminue quand le nombre des microfilaires ingérées augmente, et le nombre des microfilaires passées dans l'hémocèle ne dépasse pas une valeur limite, variable avec chaque couple Filairevecteur. Ce phénomène a été établi chez Setaria labiatopapillosa-Aedes aegypti et $\mathrm{Wu}$ - 
chereria bancrofti-Aedes aegypti, mais il existe également chez Onchocerca volvulusSimulium damnosum (Philippon et Bain, sous presse).

Nous n'avons pas trouvé de particularités anatomiques du vecteur susceptibles d'expliquer la limitation. Par contre, un ensemble de faits, issus d'analyses des résultats numériques, conduit à une hypothèse qui fait intervenir à la fois l'état physiologique et le mode de dispersion des microfilaires chez l'hôte Vertébré.

\section{Chapitre $I$}

Mise en évidence d'un nombre critique de microfilaires ingérées (seuil de limitation) au-dessus duquel le pourcentage de réussite des microfilaires diminue ; existence de microfilaires aptes et de microfilaires inaptes à traverser la paroi stomacale du vecteur.

Pour chaque couple Filaire-vecteur nous reportons individuellement les résultats numériques des dissections, puis nous groupons les Moustiques examinés par classes, en fonction du nombre de microfilaires ingérées; nous évaluons le pourcentage de microfilaires passées dans l'hémocèle pour l'effectif de chaque classe (fig. 1 à 3 ). Ceci

\begin{tabular}{|c|c|c|}
\hline M. I. & M. P. & $\%$ M.P. \\
\hline 1 & $0-1-1$ & 66,6 \\
\hline 2 & & \\
\hline 3 & $0-1$ & 33,3 \\
\hline 4 & $1-4$ & 62,2 \\
\hline 5 & $3-1-5$ & 56 \\
\hline 6 & 4 & 66,6 \\
\hline 7 & $3-4-7$ & 66,6 \\
\hline 8 & & \\
\hline 9 & $5-4-5-5$ & 52,9 \\
\hline $10-15$ & $7-11-5$ & 63 \\
\hline $16-20$ & $2-8-4-4-12-11-4-6$ & 35 \\
\hline $21-25$ & $7-0-17-13-16$ & 41,6 \\
\hline $26-30$ & 2 & 6,9 \\
\hline $31-40$ & $1-4-5$ & 9,5 \\
\hline $41-50$ & $7-14-3-2-9-12$ & 17,2 \\
\hline $51-70$ & $8-9-14-11$ & 18,5 \\
\hline $71-100$ & $11-16-16$ & 17,2 \\
\hline $100-150$ & $3-6-6-9$ & 4,8 \\
\hline $150-200$ & & \\
\hline $200-300$ & 12 & 4,8 \\
\hline
\end{tabular}

FIG. 1. - Wuchereria bancrofti, Aedes aegypti. Rapport numérique entre microfilaires ingérées (M.I.) et microfilaires passées dans l'hémocèle (M.P.) (dans la colonne de gauche, les $q$ ayant ingéré plus de 10 microfilaires sont groupées par classes de plus en plus importantes; dans la colonne du milieu, le nombre des microfilaires passées dans l'hémocèle est donné séparément pour chaque $q$ disséquée). Dans ce tableau, nous utilisons les dissections effectuées $12 \mathrm{~h}$ et $24 \mathrm{~h}$ après le repas 
permet d'exposer les variations de ce pourcentage en fonction du nombre de microfilaires ingérées.

1) W. bancrofti-A. aegypti (fig. 1).

Le pourcentage des microfilaires qui passent dans l'hémocèle reste élevé (de 40 à $60 \%$ ) quand les Moustiques prélèvent de 1 à 25 microfilaires; au-delà de 25 microfilaires ingérées, le pourcentage de réussite décroît pour atteindre des valeurs très basses de l'ordre de $5 \%$.

2) S. labiatopapillosa-A. aegypti (fig. 2).

Dans ce couple, les résultats sont moins clairement lisibles; le pourcentage de réussite des microfilaires est élevé pour les petites prises de microfilaires ; au-delà de 6-9 microfilaires ingérées, ce pourcentage décroît.

\begin{tabular}{|c|c|c|}
\hline M. I. & M. P. & $\%$ M.P. \\
\hline 1 & $1-1-1-1-1-1-0$ & 76 \\
\hline 2 & $2-2-2-2-2-2-2-2-2-2-1-1-1-1-1-1-0$ & 66 \\
\hline 3 & $2-2-2-2-1-1-1-1-3-3-1-1-1$ & 59 \\
\hline 4 & $4-2-1-1-0-4-2-1-1-0-0-2-2-0$ & 38 \\
\hline 5 & $5-3-3-4-3-1-3-2-2-1-1$ & 52 \\
\hline 6 & $4-3-1-1-1-0-5-4-1-1$ & 41 \\
\hline 7 & $1-1-1-6-2-4-2-1-0$ & 28 \\
\hline 8 & $3-3-3-3-4-4-1-3-2-1$ & 26 \\
\hline 9 & $6-4-1-5-9-2-1-1-5$ & 42 \\
\hline 10 & $2-2-2-1-1-4-4-3-1$ & 22 \\
\hline 11 & $5-1-1-4-9-3-3-3-2$ & 29 \\
\hline 12 & $2-2-2$ & 17 \\
\hline 13 & $0-3$ & \\
\hline 14 & $1-9-7-2$ & 28 \\
\hline 15 & $2-0-6-3-3$ & 18 \\
\hline $16-20$ & $9-4-2-2-0-6-5-4-4-3-1-2-0$ & 18 \\
\hline $21-25$ & $2-1-8$ & 6,5 \\
\hline $26-30$ & & \\
\hline $31-35$ & & \\
\hline $36-40$ & & \\
\hline $41-45$ & & \\
\hline $46-50$ & 12 & 25 \\
\hline $51-60$ & & \\
\hline $61-160$ & 54 & \\
\hline $161-270$ & 238 & \\
\hline
\end{tabular}

Fig. 2. - Setaria labiatopapillosa, Aedes aegypti. Rapport numérique entre microfilaires ingérées et microfilaires passées dans l'hémocèle (dans la colonne de gauche, les $q$ ayant ingéré plus de 15 microfilaires sont groupées par classes de plus en plus importantes; dans la colonne du milieu, le nombre des microfilaires passées dans l'hémocèle est donné séparément pour chaque $q$ disséquée). Dans ce tableau, nous utilisons les dissections effectuées $9 \mathrm{~h}, 12 \mathrm{~h}$ et $24 \mathrm{~h}$ après le repas. Les chiffres en italique correspondent aux passages anormaux de microfilaires à gaine dans l'hémocèle 


\begin{tabular}{|c|c|c|}
\hline M. I. & M. P. & $\%$ M. P. \\
\hline 1 & $1-0-0$ & 33,3 \\
\hline 2 & $1-0-1-1-1-0$ & 33,3 \\
\hline 3 & $1-2-0-2-2-0-0$ & 33,3 \\
\hline 4 & $\overline{0-1-0-1}$ & 15 \\
\hline 5 & $0-1-1$ & 10 \\
\hline 6 & $0-0$ & 0 \\
\hline 7 & $0-1-0$ & 4,7 \\
\hline 8 & $0-1-1$ & 8,1 \\
\hline 9 & 1 & 11,1 \\
\hline 10 & $1-1-0$ & 2 \\
\hline $11-15$ & $0-5-0-1-0$ & 9,2 \\
\hline $15-20$ & $1-0-4-2-2-0$ & 10,5 \\
\hline $21-30$ & $0-0-1-1-0-5-1-3-4-1-10-0$ & 8,6 \\
\hline $31-40$ & $0-0-1-1-0-13$ & 7,1 \\
\hline $41-50$ & $1-3-0-0-2-2$ & 2,9 \\
\hline $51-75$ & $1-1-0-0-5-10-0-0$ & 3,4 \\
\hline $76-100$ & $1-1-11-1-2$ & 3,5 \\
\hline $100-150$ & $2-3-1-2-0-1-22$ & 3,5 \\
\hline $151-200$ & $0-3-1-0-0-1-0-0-1-1$ & 0,5 \\
\hline $201-250$ & $0-2$ & 0,4 \\
\hline $251-300$ & 185 & Cas anormal \\
\hline
\end{tabular}

FIG. 3. - Onchocerca volvulus, Simulium damnosum. Rapport numérique entre microfilaires ingérées et microfilaires passées dans l'hémocèle (dans la colonne de gauche, les $q$ ayant ingéré plus de 10 microfilaires sont groupées par classes de plus en plus importantes; dans la colonne du milieu, le nombre des microfilaires passées dans l'hémocèle est donné séparément pour chaque $q$ disséquée). Dans ce tableau, nous utilisons les dissections effectuées $12 \mathrm{~h}$ après le repas

3) O. volvulus-S. damnosum (fig. 3).

Ici, le pourcentage de réussite n'est élevé (33\%) que pour d'infimes prises de microfilaires; au-delà de 3 microfilaires ingérées, ce pourcentage décroît rapidement, et pour les repas riches en microfilaires il ne dépasse pas $0,5 \%$.

Ainsi, pour ces 3 couples Filaire-vecteur, les résultats numériques montrent que la proportion des microfilaires qui passent dans l'hémocèle est élevée et constante quand le repas est pauvre en microfilaires, et qu'elle diminue au-delà d'un nombre critique de microfilaires ingérées, que nous appelons seuil de limitation. Ce seuil varie avec chaque ensemble Hôte-Filaire-vecteur étudié : il est aux environs de 3 microfilaires pour $O$. volvulus-S. damnosum, de 25 microfilaires pour $W$. bancrofti- $A$. aegypt $i$ et de 6-9 microfilaires pour $S$. labiatopapillosa- $A$. aegypti.

Selon nous, l'existence de ce seuil signifie que le donneur porte deux groupes de 
microfilaires : - les unes seraient physiologiquement aptes à traverser la paroi stomacale du vecteur (microfilaires « + ) et formeraient des populations peu denses ; les autres seraient physiologiquement inaptes à traverser la paroi stomacale (microfilaires «-») et formeraient des populations plus denses.

\section{Chapitre II}

Remarques sur la distribution de fréquence des vecteurs en fonction du nombre de microfilaires ingérées; coexistence de deux types de répartition de microfilaires chez le donneur.

Ces études de distribution sont effectuées chez les 3 couples Filaires-vecteurs présentant le phénomène de limitation, mais également, à titre de comparaison, chez $W$. bancrofti- $A$. gambiae A.

Les distributions de fréquence des $q$ en fonction du nombre des microfilaires ingérées sont représentées pour chaque couple Filaire-vecteur (fig. 4 à 7); les vecteurs

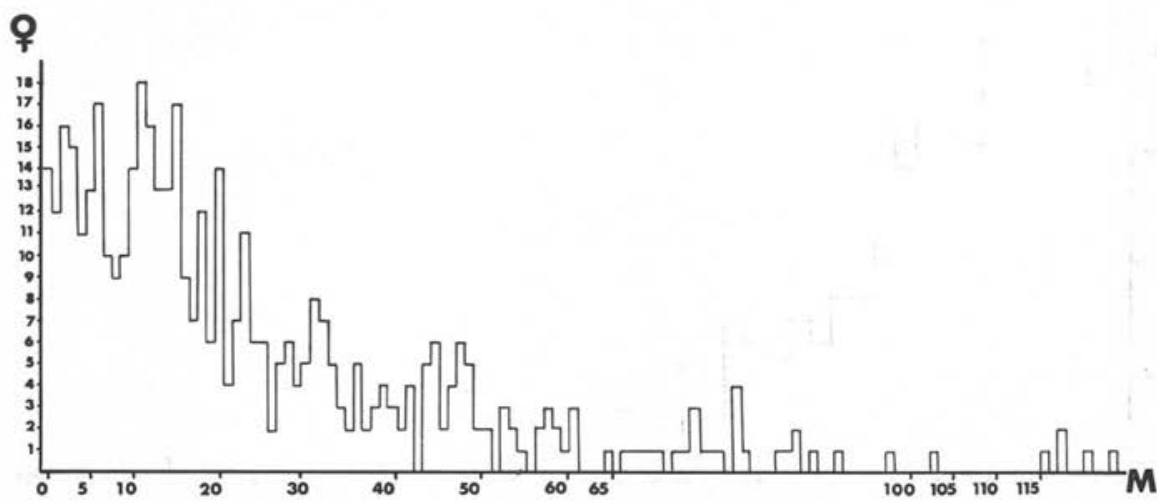

FIG. 4. - Wuchereria bancrofti, Anopheles gambiae A. Distribution de fréquence des $q$ en fonction des microfilaires ingérées (abscisses: nombre des microfilaires ; ordonnées : effectif des १). Les 4 prises suivantes de microfilaires ne sont pas représentées sur le graphique: 146; $154 ; 171 ; 175$

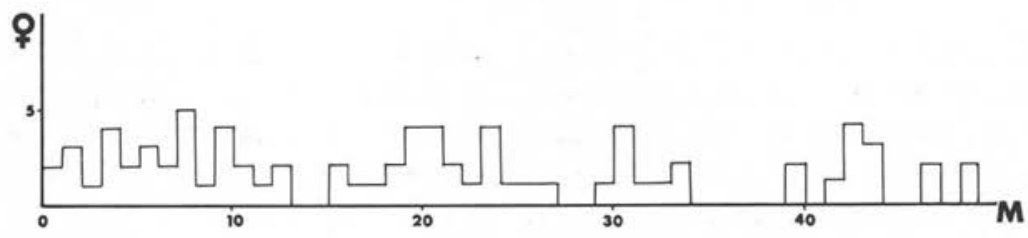

Fig. 5. - Wuchereria bancrofti, Aedes aegypti. Distribution de fréquence des $\uparrow$ en fonction du nombre des microfilaires ingérées (en abscisses, nombre des microfilaires; en ordonnées, effectif des $\$$ ) ; les 19 prises de microfilaires suivantes n’ont pas pu être placées sur l'histogramme: $62 ; 67 ; 69 ; 75 ; 77 ; 77 ; 81 ; 88 ; 96 ; 99$; $104 ; 104 ; 106 ; 108 ; 116 ; 118 ; 120 ; 141 ; 227$ 
sont groupés par classes ayant ingéré $0,1,2,3$, etc. microfilaires, sauf pour $O$. volvulus$S$. damnosum où les prises de microfilaires sont groupées de 0 à 15,16 à 30 , etc. microfilaires. Chez Wuchereria-Anopheles (fig. 4), les prises de microfilaires varient de 0 à 175 microfilaires et le mode de la distribution (sommet de l'histogramme) est environ à 12. Chez Wuchereria-Aedes (fig. 5), les prises de microfilaires varient de 0 à 227 et le mode peut être situé approximativement à 7-9 microfilaires. Chez Setaria-Aedes (fig. 6), les prises de microfilaires varient de 0 à 756 et le mode est à 2-3. Chez Oncho-

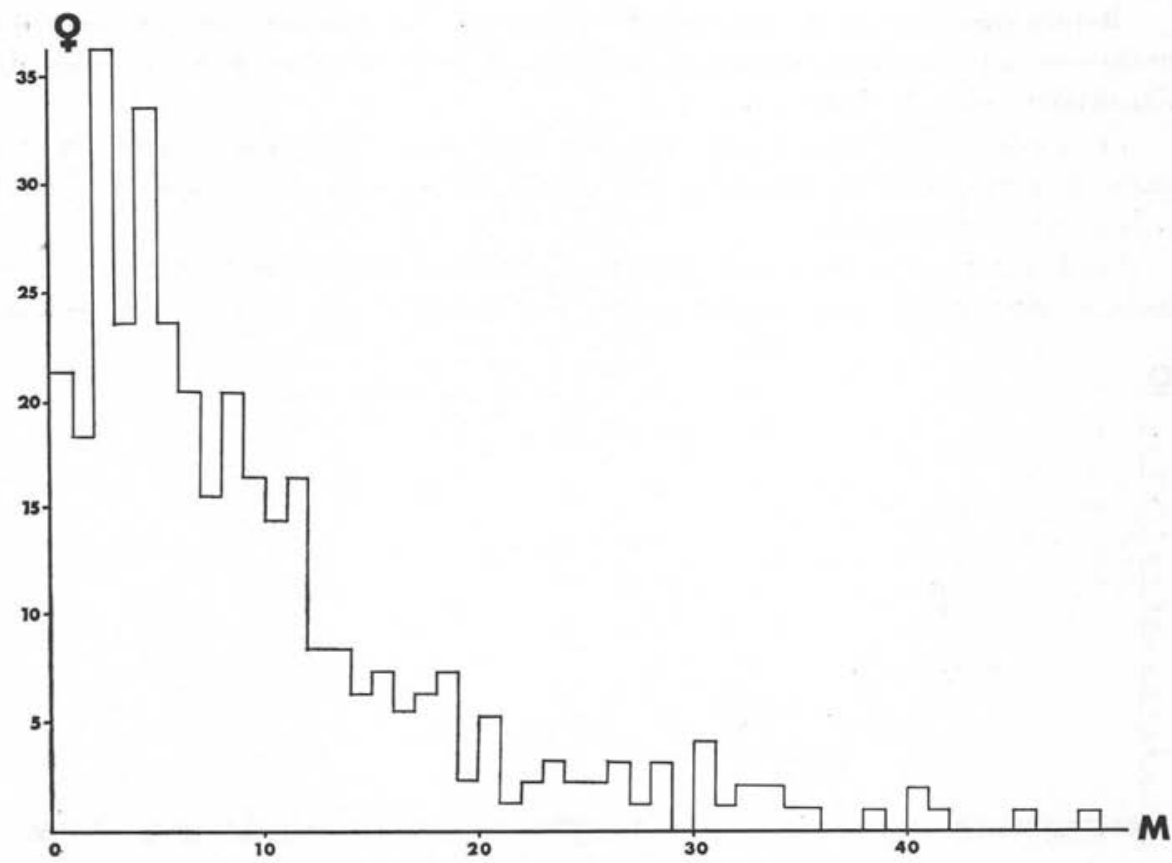

F1G. 6. - Setaria labiatopapillosa, Aedes aegypti. Distribution de fréquence des $q$ en fonction du nombre des microfilaires ingérées (abscisses: nombre des microfilaires ingérées; ordonnées : effectif des ?). Les 16 prises de microfilaires suivantes ne sont pas représentées sur le graphique : $50 ; 52 ; 53(2+) ; 55 ; 56 ; 58 ; 59 ; 73 ; 92 ; 101 ; 112 ; 115 ; 160 ; 269 ; 756$

cerca-Simulium, la figure 7 montre que les prises de microfilaires varient de 0 à 840 (résultats obtenus avec l'Onchocerquien I; cf. Philippon et Bain, sous presse); pour définir le véritable mode de la distribution de Simulium, il faut établir un deuxième histogramme dans lequel les prises de microfilaires ne sont pas groupés par classes (fig. 12); le mode réel paraît alors égal à 1 .

Dans tous les cas, ces distributions sont très étalées et montrent ainsi que les prises de microfilaires, dans des conditions expérimentales identiques, varient dans des limites considérables; ainsi une Simulie peut prendre $\mathrm{O}$ microfilaire ou un millier de microfilaires. 
Or, si l'on estime que les microfilaires sont équivalentes à des unités réparties au hasard dans un milieu homogène où les vecteurs les prélèvent, les prises de microfilaires devraient être régies par la loi de Poisson et la distribution des vecteurs devrait correspondre à une courbe de Poisson.

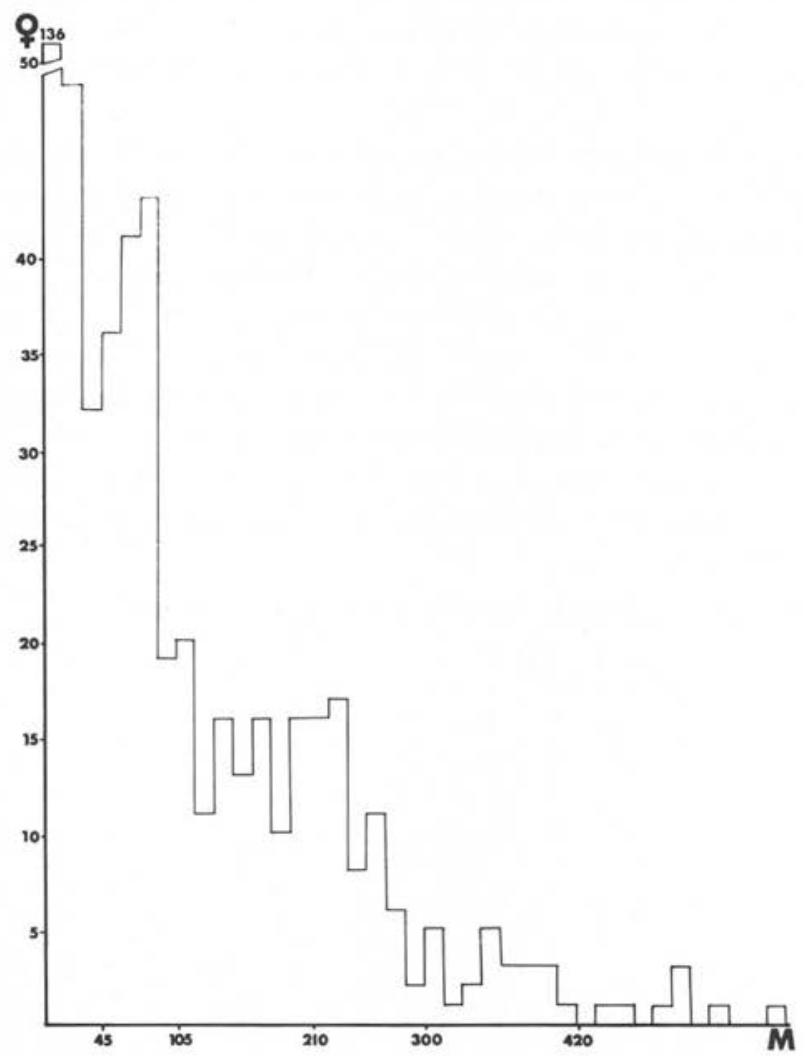

Fig. 7. - Onchocerca volvulus, Simulium damnosum. Distribution de fréquence des $q$ en fonction du nombre des microfilaires ingérées (abscisses: nombre des microfilaires ingérées groupées par classes de 15 ; ordonnées: effectif des 9). Les 4 prises de microfilaires suivantes ne sont pas représentées sur le graphique: 600 ; $622 ; 800 ; 840$

Sans tenter une analyse mathématique précise de ces distributions, nous voudrions montrer seulement que l'hypothèse de deux populations distinctes de microfilaires n'est pas incompatible avec les chiffres obtenus.

Pour représenter la courbe réelle de la distribution des $q$ en fonction du nombre des microfilaires ingérées, les effectifs des $q$ sont calculés en pourcentage de la popula- 
tion totale. Pour construire la distribution théorique de Poisson nous choisissons dans les tables un paramètre de façon à ce que la courbe théorique se calque aussi bien que possible sur les premiers points de coordonnées de la courbe réelle. Deux cas sont alors à envisager :

1) la courbe théorique coïncide bien avec le début de la courbe réelle; étant donné que cette courbe théorique est calculée pour une population de $100 \%$, ceci signifie qu'il n'y a pas de population de $q$ à distribution non poissonienne, ou qu'elle est négligeable ;

2) la courbe théorique est parallèle au début de la courbe réelle et située au-dessus d'elle ; nous constatons qu'on peut passer de la première à la seconde en transformant toutes les ordonnées théoriques de façon à ce que les nouvelles ordonnées (ou ordonnées réelles) fassent avec les précédentes un rapport constant $\mathrm{N}$

$$
\left(\mathrm{N}=\frac{\text { ordonnées réelles }}{\text { ordonnées théoriques) ; }}\right.
$$

le rapport $\mathrm{N}$ est estimé directement par lecture du graphique. Cette opération n'est pas arbitraire; elle signifie que la population qui a une distribution poissonienne ne comprend pas la totalité des $q$ mais qu'il existe une population non poissonienne importante ; elle implique également que le rapport de la population poissonienne à la population totale est égal à $\mathrm{N}$.

Les particularités de chacun des 4 couples sont les suivantes:

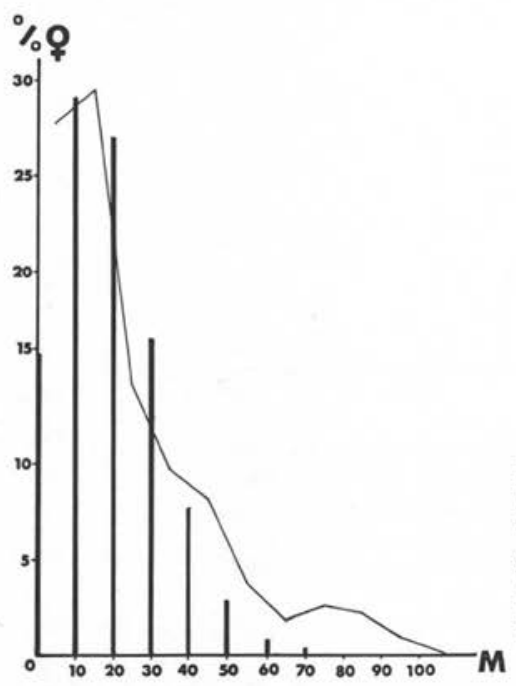

Fig. 8. - Wuchereria bancrofti, Anopheles gambiae A. En trait mince, courbe expérimentale de la distribution de fréquence des $q$ en fonction du nombre des microfilaires ingérées. En trait épais, distribution de Poisson calculée pour le paramètre 1,85. (Abscisses : nombre de microfilaires ingérées groupées par classes de 10 ; ordonnées: pourcentage des effectifs des (). Les quelques prises au-delà de 110 microfilaires ne sont pas représentées

\section{A) Wuchereria-Anopheles (fig. 8).}

La population totale est de 457 \% . Pour établir l'histogramme expérimental, ces microfilaires sont groupées par classe de 10 et l'effectif des $q$ dans chaque classe de 
microfilaires, divisé par 457, fournit la fréquence de la classe. Une distribution de P'oisson de paramètre 1,85 ajuste bien toute la partie gauche de la courbe expérimentale ; la partie résiduelle non poissonienne, située à droite sur le graphique, est ici très faible; le rapport de la population poissonienne à la population totale est d'environ $10 / 11$

B) Wuchereria-Aedes (fig. 9).

La population totale est seulement de 100 q. Notre série statistique étant traitée comme précédemment, un paramètre de 1,85 reste celui qui permet d'ajuster au mieux

Fig. 9. - Wuchereiria bancrofti, Aedes aegypti. En trait mince, courbe expérimentale de la distribution de fréquence des $q$ en fonction du nombre des microfilaires ingérées. En trait épais, distribution de Poisson, calculée pour le paramètre 1,85 . (Abscisses: nombre de microfilaires ingérées groupées bar classes de 10 ; ordonnées: pourcentage des effectifs des ?)
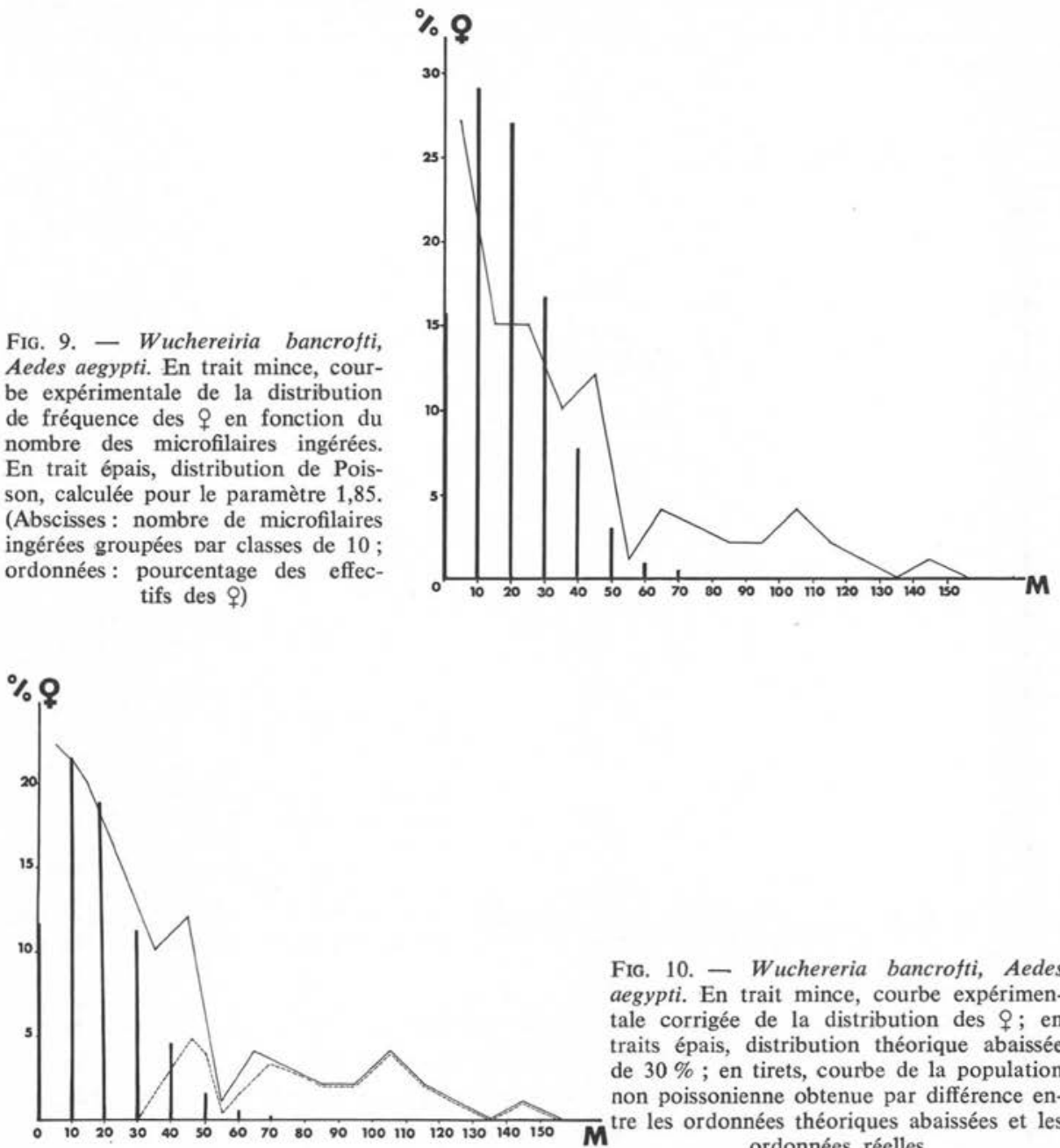

Fic. 10. - Wuchereria bancrofti, Aedes aegypti. En trait mince, courbe expérimentale corrigée de la distribution des $q$; en traits épais, distribution théorique abaissée de $30 \%$; en tirets, courbe de la population non poissonienne obtenue par différence entre les ordonnées théoriques abaissées et les ordonnées réelles 
la courbe expérimentale. Ici les données sont numériquement insuffisantes car les premiers points de coordonnées sont irréguliers; mais si l'on imagine, pour pallier cette difficulté liée au hasard, un premier chiffre un peu plus bas et le deuxième un peu plus élevé, il apparaît qu'il est nécessaire d'abaisser la courbe théorique d'environ $30 \%$ pour que la courbe théorique corrigée et début de la courbe réelle coïncident approximativement. Ceci nous amène à concevoir une population résiduelle $(=$ différence entre la surface délimitée par la courbe théorique et celle délimitée par la courbe réelle) égale à environ $30 \%$ de la population totale. Cette population résiduelle peut être figurée : ses points de coordonnées sont obtenus en effectuant la différence entre ceux de la courbe réelle et ceux de la courbe théorique corrigée ; par rapport à l'axe des abscisses, cette population débuterait ainsi aux environs de 25-30 microfilaires, aurait un maximum aux environs de 40-45 microfilaires et chuterait ensuite de façon très progressive (fig. 10).

C) Setaria-Aedes (fig. 11).

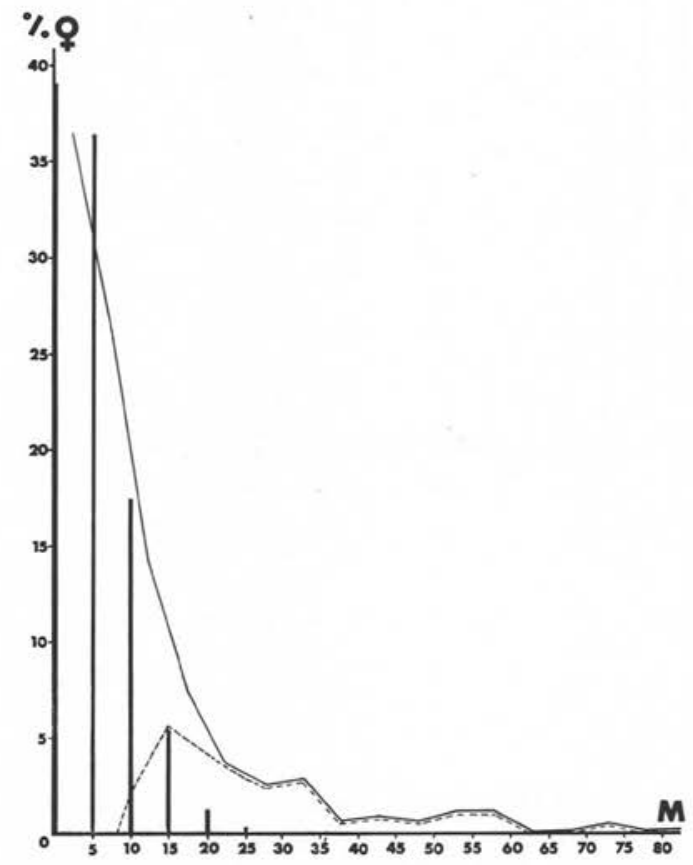

Fig. 11. - Setaria labiatopapillosa, Aedes aegypti. En trait mince, courbe expérimentale de la distribution des $q$ en fonction du nombre des microfilaires ingérées. En traits épais, distribution de Poisson pour le paramètre 0,94 . En tirets, courbe de la population non poissonienne obtenue par différence entre les ordonnées théoriques et les ordonnées réelles. (Abscisses: nombre de microfilaires ingérées groupées par classe de 5 ; ordonnées: pourcentages des effectifs des ). Les 7 prises de microfilaires comprises entre 92 et 756 microfilaires n'ont pas pu être représentées sur le graphique

La population totale est de 356 \&. Les microfilaires ingérées sont groupées par classe de 5. Une distribution ayant pour paramètre 0,94 parait ajuster assez bien le début de la courbe expérimentale. La distribution résiduelle représente le $1 / 4$ ou le $1 / 5$ environ de l'aire totale de l'histogramme de la série expérimentale. 
D) Onchocerca-Simulium (fig. 12).

La population totale est de 550 . L'histogramme, obtenu en groupant les microfilaires ingérées par 15 (fig. 7), montre que la plupart des Simulies ingèrent de 0 à 15 microfilaires. Il faut, pour préciser le sommet véritable de l'histogramme, mettre en abscisses les microfilaires par unités (fig. 11). Pour avoir une distribution de Poisson qui coïncide avec la courbe expérimentale, nous devons cette fois prendre un paramètre de 0,8 et une population de 60 environ.

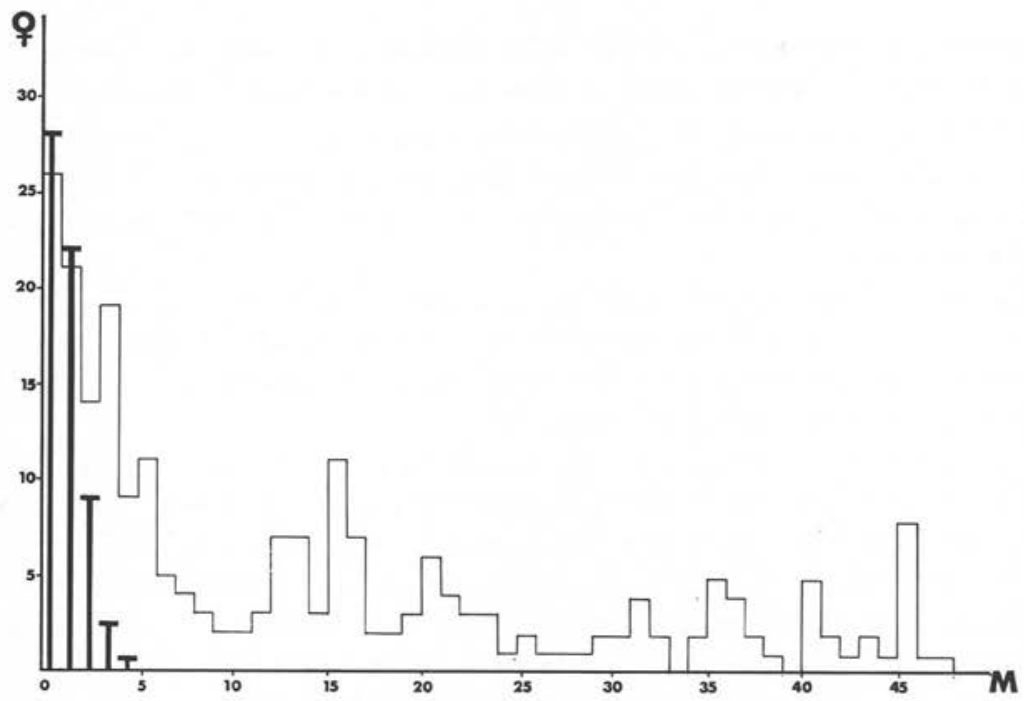

Fig. 12. - Onchocerca volvulus, Simulium damnosum. En trait mince, détail de la partie gauche de la fig. 7 représentant la distribution de fréquence des $\subsetneq$ en fonction du nombre des microfilaires ingérées. En trait épais, distribution de Poisson, calculée pour le paramètre 0,80 et pour une population de 60. (Abscisses: nombre de microfilaires ingérées; ordonnées: effectifs des †)

Contrairement aux exemples précédents, la partie gauche de la courbe, qui obéit à la distribution de Poisson, est très faible (1/9 environ) et $8 / 9$ de la population forme la courbe « résiduelle» (1).

Ainsi, quel que soit le couple Filaire-vecteur étudié, l'ensemble des $q$ gorgées se répartit en deux groupes qui révèlent l'existence, chez l'hôte définitif, de deux modes de dispersion des microfilaires: une population homogène et peu dense, correspondant à la distribution «de Poisson » des $q$, et une population hétérogène et plus dense, correspondant à la fraction «non poissonienne » des vecteurs. Ces deux ensembles de dis-

(1) Le choix de l'échelle de la fig. 12 ne permet pas de représenter toutes les prises de microfilaires; pour en avoir une vue complète, il faut se reporter à la fig 7 . 
tribution des $q$ sont schématisés fig. 13 ; l'aire de distribution poissoniénne et l'aire résiduelle se superposent sur la zone limitrophe.

Il est intéressant de constater que les deux vecteurs, Aedes et Anopheles, se gorgeant sur le même hôte wuchererien, ont une distribution poissonienne identique (même paramètre égal à 1,85$)$.

\section{Chapitre III}

Les vecteurs dont les prises de microfilaires sont comprises entre 0 et le seuil de limitation correspondent à ceux qui ont une distribution poissonienne.

Nous avons précisé au paragraphe 1 les valeurs du seuil de limitation chez les 3 couples Filaires-vecteurs qui présentent le phénomène de limitation : il est de 25 microfilaires chez Wuchereria-Aedes, de 6-9 chez Setaria-Aedes et de 3 microfilaires chez Onchocerca-Simulium.

$\mathrm{Si}$ nous reportons respectivement ces chiffres sur l'axe des abscisses des fig. 10, 11 et 12 , nous constatons qu'ils correspondent à la séparation entre la population des $q$ à distribution essentiellement poissonienne et le reste de la population, comprenant l'aire de recouvrement et la distribution résiduelle.

Cette corrélation, retrouvée 3 fois, ne peut être due au hasard; pour nous, elle signifie que la population des $q$ chez laquelle le pourcentage de réussite des microfilaires est élevé correspond à la population des $q$ qui a une distribution poissonienne (fig. 13). Ainsi les microfilaires dispersées de façon homogène semblent correspondre aux microfilaires aptes à traverser la paroi stomacale (microfilaires « $+»)$. Dans nos trois expériences, nous remarquons que ces microfilaires ont une concentration plus faible que celle des microfilaires «—», dispersées de façon hétérogène.

\section{Cbapitre IV}

La possibilité, pour un même vecteur, d'ingérer des microfilaires « + » ou des microfilaires « - pourrait s'expliquer par l'existence de deux modes de piqûre.

Les travaux de Gordon et Lumsden (1939) sur les mécanismes de piqûre des Moustiques ont montré qu'Aedes aegypti, gorgé sur Grenouille filarienne, pique dans les vaisseaux sanguins («capillary feeding») ou provoque une hémorragie localisée en lésant les capillaires («pool feeding ») ; dans ce dernier cas, le nombre des microfilaires prélevées est beaucoup plus faible.

La dualité des mécanismes de piqûre des vecteurs hématophages doit être un phénomène général : chez Aedes aegypti, par exemple, la fig. 9 sur la distribution des $q$ gorgées sur l'Homme wuchererien montre que les 3/4 des Moustiques font un type de repas sur milieu homogène et que le dernier quart de Moustiques fait un deuxième type 


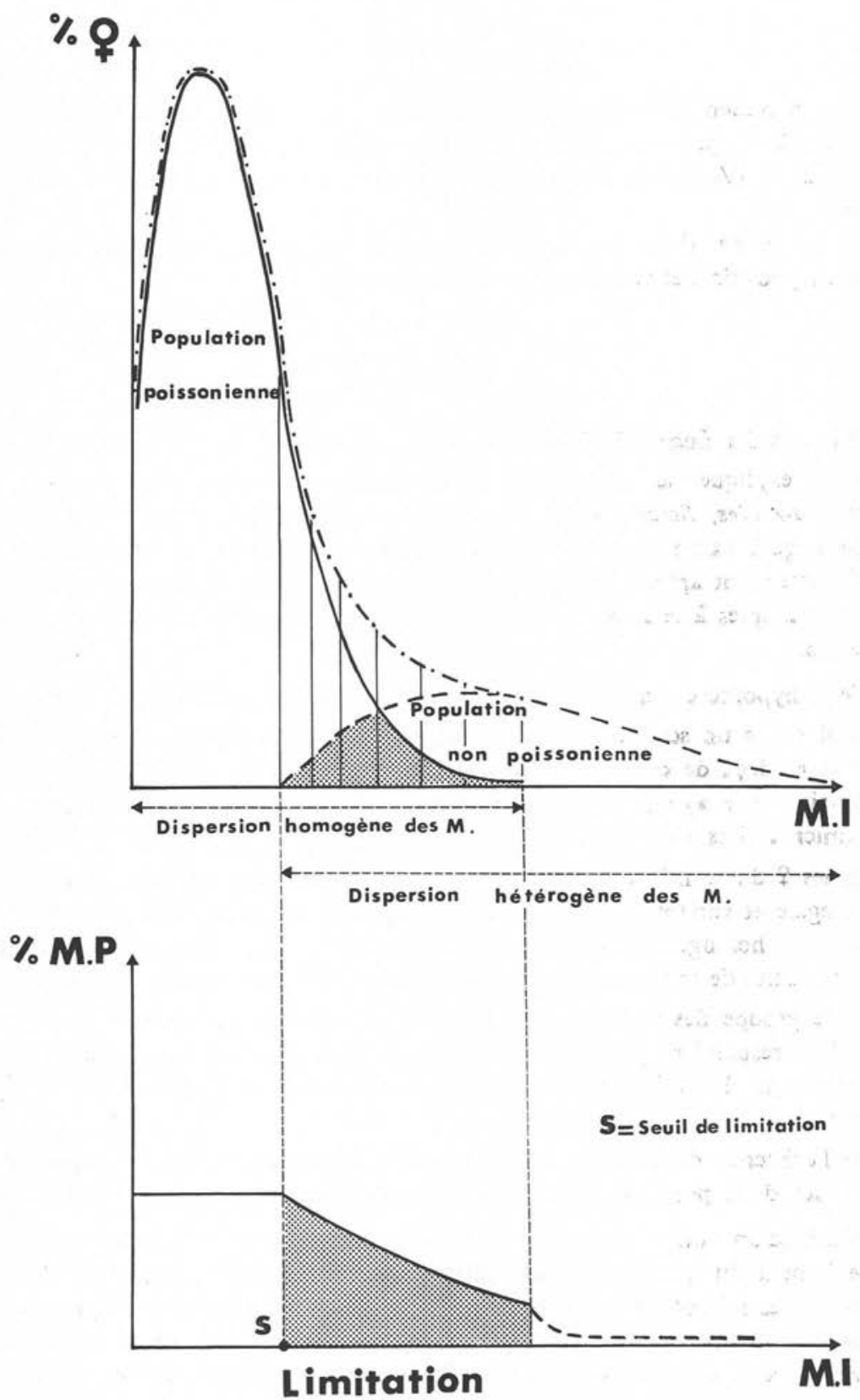

Fig. 13. - En haut: schéma représentant une courbe réelle (tirets et points) de distribution décomposée en une courbe de Poisson (trait continu) et une courbe non poissonienne (tirets); en bas : schéma représentant la variation du pourcentage de réussite des microfilaires chez les couples présentant la limitation, et ses rapports avec les 2 modes de distribution des $\$$ (M.I. $=$ microfilaires ingérées ; M.P. $=$ microfilaires passées $; \mathrm{s}=$ seuil de limitation) 
de repas sur milieu hétérogène. Nous remarquons que, sur la Vache filarienne (fig. 11), à peu près $1 / 4$ également de la population d'Aedes se gorge sur le milieu hétérogène. Dans le cas de l'Anophèle (fig. 8), la quasi-totalité des $q\left(10 / 11^{\circ}\right)$ effectue le même type de piqûre.

Le gorgement de la Simulie, tout en étant différent de celui des Moustiques, doit également pouvoir s'effectuer selon deux mécanismes.

\section{Conclusion}

\section{Mesure du degré d'adaptation Filaire-vecteur.}

Pour expliquer le phénomène de limitation, observé à trois reprises (couples Wuchereria-Aedes, Setaria-Aedes et Onchocerca-Simulium), nous avons posé comme hypothèse qu'il existe chez l'hôte vertébré deux populations de microfilaires, les unes physiologiquement aptes à traverser la paroi stomacale du vecteur, les autres physiologiquement inaptes à le faire, le vecteur prélevant les microfilaires dans l'une ou l'autre population.

Cette hypothèse semble être étayée par les faits que nous venons d'exposer :

1) il existe un seuil de limitation, c'est-à-dire un nombre critique de microfilaires ingérées: en-deçà de ce nombre, le pourcentage de réussite des microfilaires est élevé (microfilaires « + ) et, au-delà de ce nombre, le pourcentage de réussite devient très faible (microfilaires «-»);

2) les $q$ d'une même espèce de vecteur se répartissent en deux groupes, d'importance inégale et variable avec le vecteur ; un groupe qui prélève les microfilaires réparties de façon homogène (distribution poissonienne) et un groupe qui prélève les microfilaires réparties de façon hétérogène (distribution non poissonienne, résiduelle) ;

3) le groupe des vecteurs chez lequel le pourcentage de réussite des microfilaires est élevé correspond au groupe des vecteurs qui se gorgent sur la population homogène, c'est-à-dire que les microfilaires « + » correspondent aux microfilaires à répartition homogène ;

4) l'existence de deux modes de piqûre chez les vecteurs est compatible avec le fait que les deux populations de microfilaires puissent être prélevées séparément.

Ainsi, selon notre conception, le pouvoir vecteur d'un hôte intermédiaire dépend, non seulement du nombre des microfilaires ingérées, mais également de l'état physiologique de ces microfilaires (1), lié à leur mode de dispersion. Si le vecteur ne prélève que les microfilaires aptes à traverser la paroi stomacale (cas de la quasi-totalité des A. gambiae avec $W$. bancrofti), son rendement est maximum; un tel vecteur est bien adapté à la transmission de la Filaire.

(1) Il n'est pas actuellement possible de préciser la nature de ces états physiologiques ; la sénilité ou l'immaturité pourraient être des causes de l'inaptitude des microfilaires à traverser la paroi stomacale; nous pensons plutôt que cet état pourrait être réversible et se rapprocherait alors des phénomènes de périodicité des microfilaires. 
L'étude de la distribution de fréquence des vecteurs en fonction du nombre des microfilaires ingérées apporte un moyen simple de mesurer le degré d'adaptation des couples Filaires-vecteurs naturels. Chez le vecteur adapté, ne prélevant que les «microfilaires $+\gg$ (population homogène), la distribution des $q$ est poissonienne. Le couple Dipetalonema perstans-Culicoides austeni apparaît, d'après les chiffres fournis par Nicholas et Kershaw (1954), comme un exemple de bonne adaptation. Au contraire, quand le vecteur prélève surtout les «microfilaires — (population hétérogène), la majorité des $\uparrow$ a une distribution résiduelle non poissonienne ; c'est le cas du couple OnchocercaSimulium, en zone de savane guinéenne, dans l'un des foyers étudiés par Le Berre (1966).

Mais ce même couple, en forêt camerounaise, semble se comporter très différemment (chiffres fournis par Duke et Lewis, 1964): le rendement des microfilaires ingérées est plus élevé qu'en Haute-Volta (Philippon et Bain, sous presse) et, apparemment, il n'y a pas de seuil de limitation.

L'adaptation de la Filaire au vecteur qui est le résultat d'une suite de sélections, atteint donc des degrés variables pour un même couple selon les localisations géographiques.

\section{Bibliographie}

Bain (O.) et Brengues (J.), sous presse. - Transmission de la Wucheriose et de la Setariose bovine: étude histologique de la traversée de la paroi stomacale d'Anopheles gambiae A et d'Aedes aegypti par les microfilaires. Ann. Parasitol.

Brengues (J.) et BaIn (O.), sous presse. - Passages des microfilaires de l'estomac vers l'hémocèle du vecteur dans les couples Wuchereria bancrofti - Anopheles gambiae A, W. brancrofti - Aedes aegypti et Setaria labiatopapillosa - A. aegypti. Cah. O.R.S.T.O.M., série Ent.

DUKE (B. O. L.) et LEwIS (D. J.), 1964. - Studies on factors influencing the transmission of Onchocerciasis. III. Observations on the effect of the peritrophic membrane in limiting the development of Onchocerca volvulus microfilariae in Simulium damnosum. Ann. trop. Med. Parasit., 58, 83-88.

Gordon (R. M.) et LUMSDEN (W. H. R.), 1939. - A study of the behaviour of the mouth parts of mosquitoes when taking up blood from living tissue; together with some observations of the ingestion of microfilariae. Ann. trop. Med. Parasit., 33, 259-278.

LE Berre (R.), 1966. - Contribution à l'étude biologique et écologique de Simulium damnosum, Theobald 1903 (Diptera, Simuliidae). Mémoire O.R.S.T.O.M., 17, 204 pp.

Nicholas (W.-Z.) et Kershaw (W. E.), 1954. - Studies on the intake of microfilariae by their insect vectors, their survival, and their effect on the survival of vectors III. The intake of the microfilariae of Acanthocheilonema perstans by Culicoides austeni and C. grahami. Ann. trop. Med. Parasit., 48, 200-206.

Philippon (B.) et BAIN (O.), sous presse. - Transmission de l'Onchocercose humaine en zone de Savane d'Afrique Occidentale. Passage des microfilaires d'Onchocerca volvulus Leuck. dans l'hémocèle de la femelle de Simulium damnosum Th. Cah. O.R.S.T.O.M., série Ent. 